\title{
Pre-Operative Imaging Assessment is Mandatory Prior Structural Transcatheter Procedures: a Case of Minimally Invasive Bail-Out Sapien XT Implantation in Mitral Position
}

\author{
Stefano Salizzoni ${ }^{1}$, Giovanni Marchetto ${ }^{1}$, Matteo Marro ${ }^{1 *}$, Andrea Baronetto ${ }^{1}$, Marcella Jorfida ${ }^{2}$, Maurizio \\ D’Amico $^{2}$ and Mauro Rinaldi ${ }^{1}$
}

${ }^{1}$ Department of Surgical Sciences, Città della Salute e della Scienza di Torino, University of Turin, Italy

${ }^{2}$ Department of Medical Sciences, Città della Salute e della Scienza di Torino, University of Turin, Italy

Submission: December 19, 2017; Published: February 20, 2018

*Corresponding author: Matteo Marro, Division of Cardiac Surgery, Department of Surgical Sciences, Città della Salute e della Scienza di Torino, University of Turin, Corso Bramante 88, 10126 Torino, Italy, Tel: +39-011-6335511; Fax: +39-011-6336130; Email: matteo.marro1@gmail.com

\section{Abstract}

Degeneration of bioprosthesis may occur in elderly and frail patients with an extremely high risk for redo cardiac surgery. Transcatheter valve-in-valve procedures represent a valuable treatment option in high-risk patients [1,2]. However incidental findings may contraindicate the transcatheter procedure. We describe the original case of a patient who was scheduled for transapical mitral valve-in-valve, but due to a left atrial mass underwent mini-invasive surgery with successful direct mitral valve-in-valve delivery and excision of the mass.

Keywords: TAVI; Transcatheter; Mitral; Valve-in-valve; Bioprosthesis failure; Minimally invasive surgery

\section{Introduction}

Degeneration of bioprosthesis may occur in elderly and frail patients with an extremely high risk for redo cardiac surgery. Transcatheter valve-in-valve procedures represent a valuable treatment option in high-risk patient population [1-3]. Usually before transcatheter valve procedures, patients are studied with CT-scan and/or transesophageal echocardiography in order to estimate the exact anatomical sizes.

\section{Case Report}
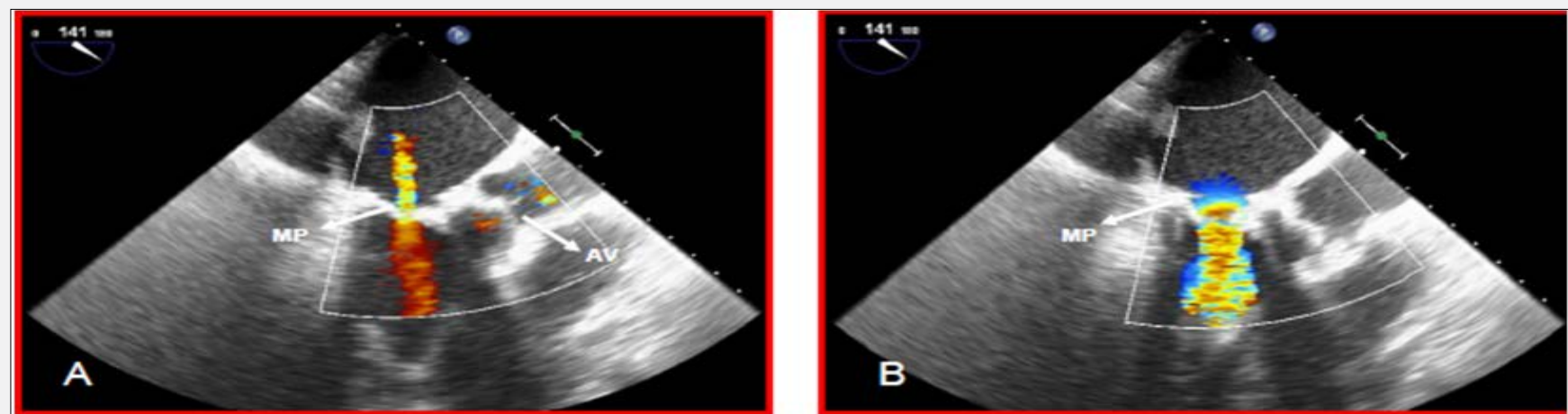

Figure 1: A. Transthoracic echocardiography showing mitral prosthesis (MP) regurgitation during systole B. Transthoracic echocardiography showing turbulent flow due to mitral prosthesis (MP) stenosis during diastole.

A 82-years old man with rheumatic heart disease in 2007 underwent mitral valve replacement with a $27 \mathrm{~mm}$ Carpentier Perimount bioprosthesis (Edwards Life sciences, Irvine, CA, USA) via sternotomy. In January 2015 the patient was admitted for syncope with dizziness and vomiting and worsening of shortness of breath; CT head scan, ear-nose-throat evaluation and Holter ECG were normal. Transthoracic Echocardiography (TTE) showed a $43 \%$ left ventricle ejection fraction with severe mitral stenosis 


\section{Journal of Cardiology \& Cardiovascular Therapy}

(mean gradient $14.5 \mathrm{mmHg}$ ) and moderate regurgitation, mild tricuspid regurgitation with $69 \mathrm{mmHg}$ of Systolic Pulmonary Artery Pressure (Figure 1 and Video 1).

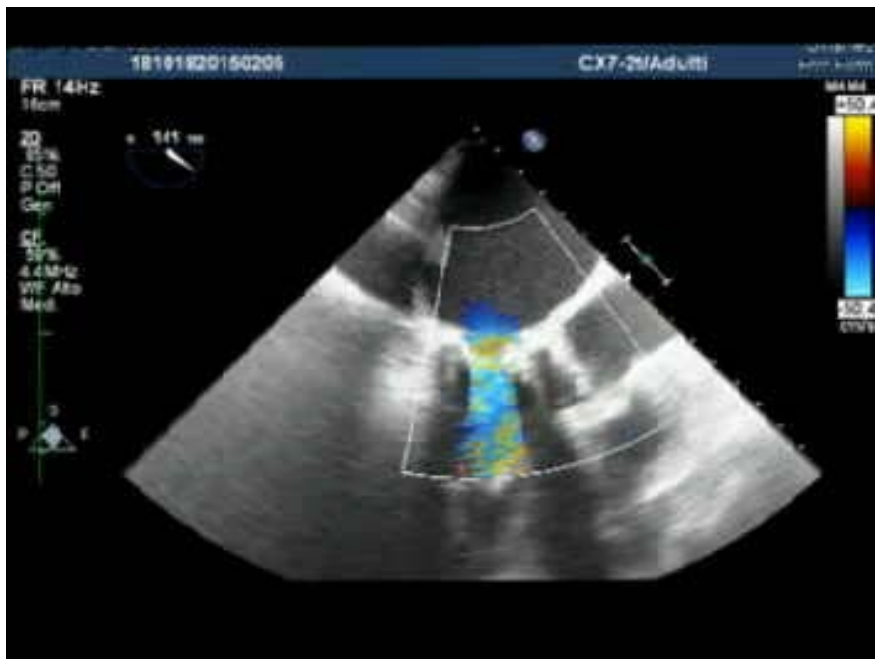

Video 1: Pre-operative transthoracic echocardiography showing mitral prosthesis (MP) regurgitation during systole and turbulent flow due to mitral prosthesis stenosis during diastole.

Because of age and frailty of the patient, and the history of previously implanted bioprosthesis, a trans-apical mitral valve-invalve procedure was indicated. Since we knew the precise size of mitral prosthesis, we did not performed any usual imaging (CTscan or TEE) to estimate the valvular size.

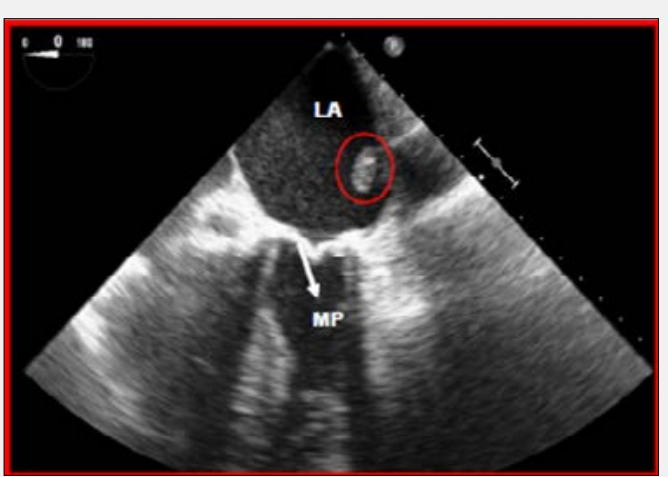

Figure 2 : Transesophageal intraoperative echocardiography showing left atrial (LA) mass (circled by the red line) and the mitral prosthesis (MP).

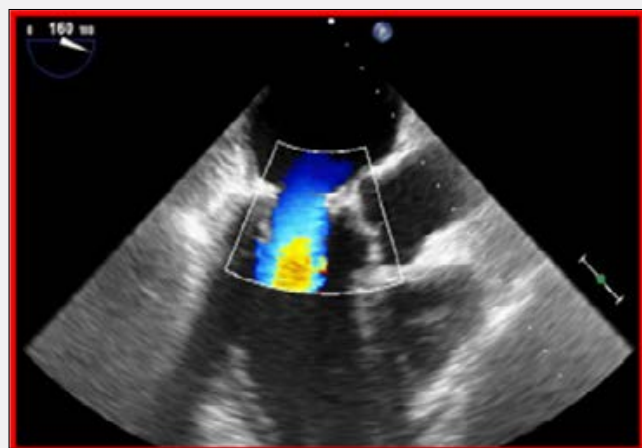

Figure 3 : Post procedure transesophageal echocardiography showing regular position of mitral prosthesis and laminar flow during systole. Left atrial mass disappered.
Unexpectedly intra-operative transesophageal echocardiography revealed the presence of a sessile and compact formation of $2.5 \times 1 \mathrm{~cm}$ situated between the roof of the left atrium and the atrial septum (Figure 2 and Video 2) that was not detectable on transthoracic echocardiography. Because of the risk of embolization the procedure was switched to open heart surgery through a right anterolateral minithoracotomy at the fourth intercostal space [4]. Cardio pulmonary by-pass through the right femoral artery, the right femoral vein and right jugular vein was established. Under ventricular fibrillation the left atrial mass was quickly removed and a 29mm Sapien XT (Edwards Life sciences, Irvine, CA, USA), mounted on the trans apical delivery system, was easily implanted under direct vision inside 27-mm Carpentier Perimount bioprosthesis. Intraoperative transesophageal echocardiography showed normal transvalvular gradients and a laminar flow (Figure 3 and Video 3 ). The histologic exam of the mass confirmed the hypothesis of mixoma. The patient was discharged on 6th post-operative day. The one year echocardiographic follow-up is comparable to the one at discharge (Video 4).

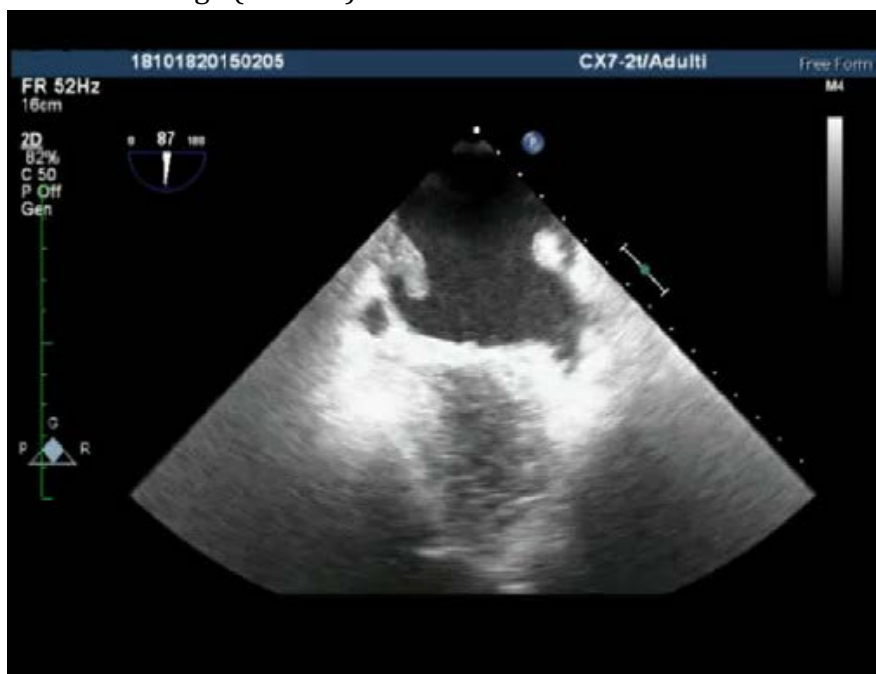

Video 2: Transesophageal intraoperative echocardiography showing left atrial (LA) mass.

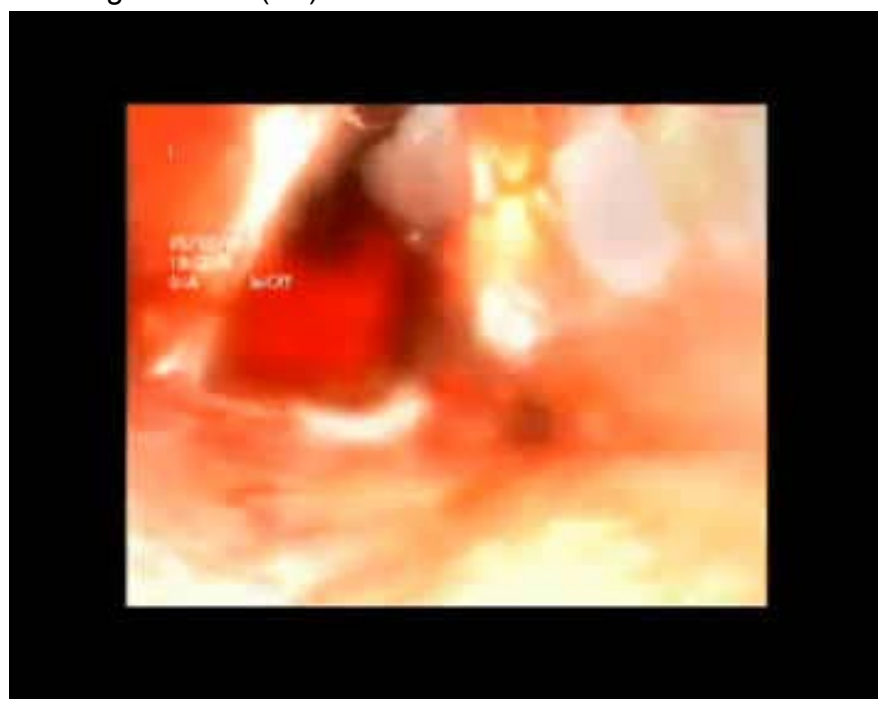

Video 3: Sapien XT, mounted on the transapical delivery system, was easily implanted under direct vision inside mitral bioprosthesis. 


\section{Journal of Cardiology \& Cardiovascular Therapy}

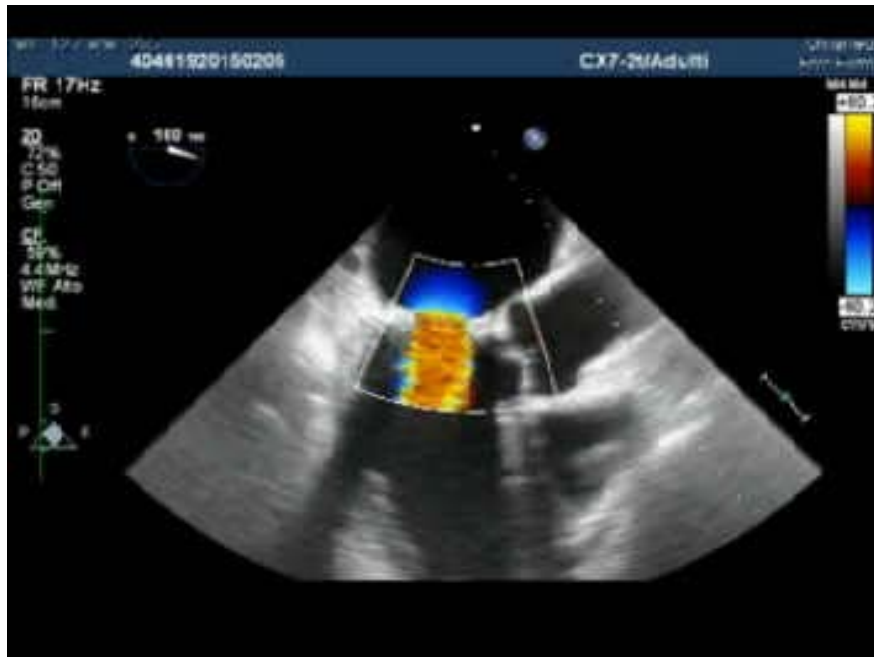

Video 4: Post procedure transesophageal echocardiography showing regular position of mitral prosthesis and laminar flow during systole. Left atrial mass disappered.

\section{Conclusion}

Valve-in-valve implantation in mitral position is becoming an increasingly accepted treatment option for patients deemed unsuitable for redo operation [5-8].

This case demonstrates:

a) The importance of pre-operative imaging assessment (CT thoracic scan and/or transesophageal echocardiography) prior of any structural transcatheter intervention, even if exact anatomical sizes are known; it is not unusual to find other unexpected anatomical surprises.

b) The easiness of direct mitral valve-in-valve procedure, that to date has been described only once [5]. c) The efficacy of the procedure after one year.

\section{References}

1. Cheung A, Webb JG, Wong DR, Ye J, Masson JB, et al. (2009) Transapical transcatheter mitral valve-in-valve implantation in a human. Ann Thorac Surg 87(3): e18-e20.

2. Cheung A, Webb JG, Barbanti M, Freeman M, Binder RK, et al. (2013) 5-year experience with transcatheter transapical mitral valve-in-valve implantation for bioprosthetic valve dysfunction. J Am Coll Cardiol 61(17): 1759-1766.

3. McElhinney DB, Cabalka AK, Aboulhosn JA, Eicken A, Boudjemline $Y$, et al. (2016) Transcatheter tricuspid valve-in-valve implantation for the treatment of dysfunctional surgical bioprosthetic valves: an international multicenter registry study. Circulation 133(16): 15821593.

4. Marchetto G, D'Armini AM, Rinaldi M, Viganò M (2005) Portaclamp in video-assisted minimally invasive cardiac surgery: surgical technique and preliminary clinical experience. Eur J Cardiothorac Surg 27(6): 1122-1124.

5. Mick SL, Roselli EE, Kapadia S, Tuzcu EM, Krishnaswamy A, et al. (2016) Postoperative migration of an edwards-sapien xt mitral valvein-valve treated with direct vision implantation during beating-heart bypass. Ann Thorac Surg 101(3): 1182-1185.

6. Seiffert M, Conradi L, Baldus S, Schirmer J, Knap M, et al. (2012) Transcatheter mitral valve-in-valve implantation in patients with degenerated bioprostheses. JACC Cardiovasc Interv 5(3): 341-334.

7. Cerillo AG, Chiaramonti F, Murzi M, Bevilacqua S, Cerone E, et al. (2011) Transcatheter valve in valve implantation for failed mitral and tricuspid bioprosthesis. Catheter Cardiovasc Interv 78(7): 987-999.

8. Salizzoni S, Barbero C, Grosso Marra W, Moretti C, Rinaldi M (2014) Transapical implantation of an Edwards SAPIEN XT in a degenerated mitral bioprosthesis without fluoroscopic landmarks. J Card Surg 29(5): 625-627.

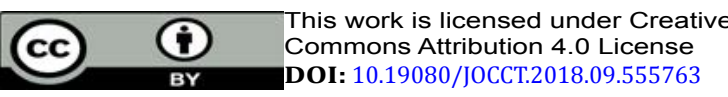

